\title{
Down Syndrome and Congenital Heart Disease: Single centre, Prospective Study
}

\author{
Shrestha $M,^{1^{*}}$ Shakya $U^{1}$ \\ ${ }^{1}$ Paediatric Cardiology Unit, Department of Cardiology, Shahid Gangalal National Heart \\ Centre, Bansbari, Kathmandu, Nepal
}

\author{
*Corresponding Author: \\ Dr. Manish Shrestha, \\ Paediatric Cardiology Unit, \\ Department of Cardiology, \\ Shahid Gangalal National Heart Centre, \\ Bansbari, Kathmandu, Nepal \\ E-mail: manishrestha@msn.com
}

\section{Citation}

Shrestha M, Shakya U. Down Syndrome and Congenital Heart Disease: Single centre, Prospective Study. Nepal Journal of Medical Sciences. 2013;2(2):96-101.

\begin{abstract}
Background: The objective of this study was to evaluate the children with down syndrome regarding the frequency and types of congenital heart disease and associated pulmonary hypertension.

Method: A prospective study was carried out to all the children with down syndrome visited in pediatric outpatient department over the period of one year. Necessary data were recorded in preformed format.

Results: Fifty children with down syndrome were evaluated. Forty (80\%) had an associated congenital heart disease. The median age at diagnosis was 2 years (range $=4$ days to 12 years). In 26 patients $(65 \%)$, the cardiac lesion was isolated, while 14 patients $(35 \%)$ had multiple defects. The most common single defect was ventricular septal defect (VSD), found in 9 of the 40 patients $(22.5 \%)$, followed by atrioventricular septal defect (AVSD) in $15 \%$, atrial septal defect (ASD) and patent ductus arteriosus (PDA) each in $10 \%$. The most frequent concomitant malformation found co-existing with other congenital cardiac lesions was PDA (15\%). Pulmonary hypertension was found in 21 of 40 patients $(52.5 \%)$ and AVSD was most frequently associated with pulmonary hypertension.

Conclusion: Congenital heart disease is very common in patient with down syndrome. VSD is the most common cardiac defect and AVSD is second to VSD. Patient with down syndrome with CHD frequently develop pulmonary hypertension at younger age hence early cardiac screening by echocardiography in these patients is crucial. Early diagnosis and management is the key to avoid irreversible hemodynamic consequences of the defect.
\end{abstract}

Keywords: Congenital heart disease; down syndrome; pulmonary hypertension

\section{Background:}

Down syndrome (DS), also known as Trisomy 21, is the most frequent chromosomal aberration associated with mild to moderate mental disability, a characteristic facial appearance, and poor muscle tone in infancy. ${ }^{1}$ The incidence of DS in live births is approximately 1 in 733, however this rate varies according to the mother's age; in mothers 45 years of age or older, the incidence reaches one in every 30 live births. After mother 35 years, the risk of conceiving a child with DS increases significantly, however younger women though having a lower risk represent half of all mothers with babies with DS because of their higher overall birth rate. ${ }^{2}$ The risk of recurrence is $1 \%$ in the general population. ${ }^{3}$

Children with DS have typical physical phenotype of mental 
retardation, brachycephaly, hypotonia, flat facies, slanted palpebral fissures, epicanthic folds, brush field spots on the iris, relatively large protruding tongue, small low set ears and hand anomalies (short and broad hand, clinodactyly of the fifth finger, Simian crease). ${ }^{4-6}$ Congenital heart defects, seizures, strabismus, nystagmus, atlantoaxial instability, cryptorchidism, increased risk of leukemia, dementia, duodenal atresia and hypothyroidism are usually associated with DS. Although there is variability in the clinical features, the constellation of phenotypic features is fairly consistent and permits clinical recognition of trisomy $21 .^{2,4-6}$

Congenital heart disease (CHD) is, without a doubt, the main factor contributing to a favorable or unfavorable course in these patients. Among all cases of CHD, 4-10\% are associated with DS, and $40-60 \%$ of DS patients present CHD. ${ }^{2,3,7} \mathrm{CHD}$ is the greatest cause of death in patients with DS during the first two years of life. ${ }^{8}$ The cardiac lesions can be single or multiple. Common CHDs are ventricular septal defect (VSD), atrial septal defect (ASD), atrioventricular septal defect (AVSD), patent ductus arteriosus (PDA) and tetralogy of Fallot (TOF). ${ }^{2}$ These children are frequently associated with pulmonary hypertension which is also one of the determining factor for the management of CHD.

Nowadays, almost all CHD in DS patients are surgically manageable, with good results. Early diagnosis and treatment is the key to achieve such results. It is recommended that DS patients should be screened by birth by pediatric cardiologist. ${ }^{9}$ However it is not possible and feasible to get screening by birth in the country like Nepal where still the home delivery is almost equal to or more than hospital delivery. Furthermore the facility of echocardiography is also not available everywhere. Shahid Gangalal National Heart Centre (SGNHC) is the first ever hospital in Nepal with full-fledged pediatric cardiology and congenital cardiac surgery facilities, and with the provision of Children Assisted Program (CAP), maximum CHD cases visited or are referred here from all over Nepal. As the association of CHD in DS is so common and it also determines the outcome (morbidity and mortality) of patients, the study is carried out to find out the spectrum of CHDs in DS patients visited here.

The objective of this study is to evaluate the children with down syndrome regarding the frequency and types of congenital heart disease and associated pulmonary hypertension.

\section{Methods:}

A prospective study was carried out to all the children with down syndrome visited in pediatric cardiology outpatient department (OPD) at SGNHC over the period of one year from October 2011 to September 2012. Down syndrome was diagnosed clinically with their typical features and verified by two pediatricians. Patients with dysmorphic features other than Down syndrome were excluded from the study. Karyotyping was done whenever feasible. Most of the cases were referred by personal pediatricians for cardiac evaluation. Detailed history and physical examination of every DS child were carried out by the author himself. The exact age of mother, eldest sibling and immediate elder sibling were recorded. The Physical findings to look in these children include: mongoloid facies, brachycephaly, depressed nasal bridge, protruding tongue, small low set ears, upward slanted eyes with epicanthal fold, short neck, short and broad hands, transverse single palmar crease, hypotonia and delayed milestones. ECG, chest x-ray and echocardiography (2-D and Doppler) were done. ECG of small DS child could not be evaluated properly either due to their uncooperativeness or due to motion artifacts so ECG evaluation was excluded from the study. Echocardiographies of all children were performed by one pediatric cardiologist using GE Vivid-7. As hypothyroidism was frequently associated with down syndrome, thyroid function test (TFT) was also included in the study. All the collected data were entered into and analysed by SPSS statistical software (version 16). Descriptive statistics (mean, median and proportion) were obtained.

\section{Results:}

There were 50 children with Down syndrome who attended OPD (1.15\% of total), and among these $40(80 \%)$ had some form of congenital heart disease. The median age at diagnosis was 2 years (range, 4 months to 12 years). The male-tofemale ratio was 1:1.4. The median age of mother was 29.5 years (range, 17 to 44 years) and $80 \%$ were younger than 35 years of age at the time of birth (i.e., $\leq 25$ years $=44 \%$ and 26 to 35 years $=36 \%$ ).

A total of 17 patients (34\%) were first-born children. Those DS children who were not first-born, age-gap was calculated between him/her and immediate elder. The median agegap was 5 years (range, 2 to 18 years). Family history was positive in $4(8 \%)$ patients.

Regarding clinical profiles, cyanosis and clubbing were present in $5(10 \%)$ and $4(8 \%)$ patients respectively. 11 $(22 \%)$ patients had normal heart sound without murmur. Second heart sound (S2) was loud in 17 (34\%) patients and single in $4(8 \%)$ patients. $39(78 \%)$ patients had murmur.

Chest X-ray revealed 25 (50\%) patients had cardiomegaly. 
Thyroid Function test revealed 12 (24\%) had hypothyroidism.

Table 1: The Frequency and types of Congenital heart disease

\begin{tabular}{lc}
\hline Cardiac lesions & Patients, $\mathbf{n = 4 0 ( \% )}$ \\
\hline Single lesion & $26(65 \%)$ \\
VSD & $9(22.5 \%)$ \\
AVSD & $6(15 \%)$ \\
ASD & $4(10 \%)$ \\
PDA & $4(10 \%)$ \\
TOF & $3(7.5 \%)$ \\
Multiple lesions & $14(35 \%)$ \\
PDA associated lesions & $6(15 \%)$ \\
ASD associated lesions & $5(12.5 \%)$ \\
PS associated lesions & $2(5 \%)$ \\
MVP associated lesions & $1(2.5 \%)$ \\
\hline
\end{tabular}

(TOF: Tetralogy of Fallot; PS: pulmonary stenosis; MVP: mitral valve prolapse)

Of the overall number of patients with CHD, 26 (65\%) had a single cardiac lesion, whereas the remaining $14(35 \%)$ had multiple defects. The most common single defect was ventricular septal defect (VSD), which was found in 9 $(22.5 \%)$ patients, followed by atrioventricular septal defect (AVSD) in $6(15 \%)$ patients, atrial septal defect (ASD) and patent ductus arteriosus (PDA) each in $4(10 \%)$ patients (Table 1).

Among the patients with multiple cardiac lesions, PDA was the most frequent associated lesions, which was found in 6 $(15 \%)$ patients followed by ASD in $5(12.5 \%)$ patients.

Table 2: Congenital heart disease and associated pulmonary hypertension

\begin{tabular}{lc}
\hline CHD & Patients, $\mathbf{n}=\mathbf{4 0}(\mathbf{\%})$ \\
\hline CHD with no PAH & $19(47.5 \%)$ \\
CHD with PAH & $21(52.5 \%)$ \\
Single lesion with PAH & $13(32.5 \%)$ \\
VSD, PAH & $5(12.5 \%)$ \\
AVSD, PAH & $5(12.5 \%)$ \\
PDA, PAH & $2(5 \%)$ \\
ASD, PAH & $1(2.5 \%)$ \\
Multiple lesions with PAH & $8(20 \%)$ \\
VSD + PDA & $5(12.5 \%)$ \\
VSD + ASD & $3(12.5 \%)$ \\
\hline
\end{tabular}

Of the 40 CHD patients with DS, 21(52.5\%) had pulmonary artery hypertension (PAH) (Table 2). PAH was found to be associated with both single and multiple cardiac lesions. VSD and AVSD are the most common single lesion associated with $\mathrm{PAH}$, each in $5(12.5 \%)$ patients, however PAH was associated in 5 out of $9(55.55 \%)$ VSD cases and 5 out of $6(83.33 \%)$ AVSD cases (Table 3). Among the multiple cardiac lesions, PDA was the most frequent coexistent lesion associated with $\mathrm{PAH}$ which was found in $5(12.5 \%)$ patients.

Table 4 compares frequency of CHD and PAH with respect to age. Proportion of PAH to CHD was also calculated.

Table 3: Proportion of PAH to Individual CHD

\begin{tabular}{llll}
\hline CHDs & Frequency & PAH & $\%$ \\
\hline VSD & 9 & 5 & 55.55 \\
AVSD & 6 & 5 & 83.33 \\
PDA & 4 & 2 & 50 \\
ASD & 4 & 1 & 25 \\
\hline
\end{tabular}

Table 4: Frequency of CHD and PAH with respect to age and proportion of $\mathrm{PAH}$ to $\mathrm{CHD}$

\begin{tabular}{llll}
\hline \multicolumn{1}{r}{ Age range (years) } & CHD & PAH & \multicolumn{1}{c}{$\%$} \\
\hline$<1$ & 12 & 8 & 66.67 \\
$1-5$ & 20 & 9 & 45 \\
$5-10$ & 5 & 3 & 60 \\
$>10$ & 3 & 1 & 33.33 \\
Total & 40 & 21 & 52.5 \\
\hline
\end{tabular}

\section{Discussion:}

Fifty children with down syndrome attended paediatric opd over the period of one year. All were included in the study. Out of them, $40(80 \%)$ had congenital heart disease which is high with respect to the world literature (40-60\%). This variation may be due to the present study is a single centre study; the enrolled children are brought by parents either for their symptoms or they are being referred by personal pediatrician suspecting heart disease or for cardiac screening. Had the sampling been taken from the place like birthing centre and all DS children been screened for cardiac defect, the incidence could have come in the range as in the literature. In the similar study in Sudan ${ }^{10}$ only $9 \%$ of all DS children have normal echocardiogram.

Down syndrome is associated with maternal ages at the extremes of the childbearing period, however our study showed it is more frequent in young mothers as they have overall high birth rate. The finding is similar to studies by Karen Summar, et $\mathrm{al}^{2}$ and De Rubens Figueroa J, et al. ${ }^{3}$ 
The present study found among 40 CHD patients, 65\% had isolated cardiac defect, which is similar to the study in libya, ${ }^{7}$ however it is different as compared to $80 \%$ in Guatemala, ${ }^{8} 74 \%$ in Mexico, ${ }^{3} 78 \%$ in Turkey. ${ }^{11}$ VSD was the most common isolated defect and was found in 9 of the 40 patients $(22.5 \%)$, which was followed by AVSD $(15 \%)$ then ASD (10\%) and PDA (10\%). These findings are consistent with the study in Asian countries; China ${ }^{12}$ and Saudi Arabia, ${ }^{13}$ however in the study in India ${ }^{14}$ ASD was more common than AVSD. The study in Guatemala ${ }^{8}$ also had the similar findings; VSD being the most common followed by ASD and AVSD. These findings differ if we compared with the study in Italy, ${ }^{15} \mathrm{USA}^{16}$ and Sudan ${ }^{10}$ where AVSD was the most common isolated congenital cardiac defect followed by VSD and then ASD or PDA (Table 5). However in the study in Mexico ${ }^{3}$ and Libya, ${ }^{7}$ ASD was the most common followed by VSD and AVSD or PDA. TOF is the most common cyanotic congenital heart disease. Other complex CHDs are rare in children with DS. These findings are consistent with the literature (Table 5). Table 5 compares the findings of different studies with the present study. The frequency and distribution of CHD in DS vary in different geographical regions, the reason for this difference is not clear, although some consistency is observed between certain global areas. This variation in geographical distribution may be caused by numerous factors, one of which could be the genetic make-up of each nation or global region; or it could be due to specific embryological mechanisms.

Table 5: Comparison of CHDs in DS patients $(n=50)$ among different countries.

\begin{tabular}{lccccccc}
\hline \multirow{2}{*}{ Cardiopathy } & $\begin{array}{c}\text { VSD } \\
\%\end{array}$ & $\begin{array}{c}\text { AVSD } \\
\%\end{array}$ & $\begin{array}{c}\text { ASD } \\
\%\end{array}$ & $\begin{array}{c}\text { PDA } \\
\%\end{array}$ & $\begin{array}{c}\text { TOF } \\
\%\end{array}$ & $\begin{array}{c}\text { CoA } \\
\%\end{array}$ & $\begin{array}{c}\text { others } \\
\%\end{array}$ \\
\hline Present study & $\mathbf{2 2 . 5}$ & $\mathbf{1 5}$ & $\mathbf{1 0}$ & $\mathbf{1 0}$ & $\mathbf{7 . 5}$ & $\mathbf{0}$ & $\mathbf{3 5}$ \\
India $^{14}$ & 26 & 3 & 12 & 5 & 16 & 0 & 39 \\
China $^{12}$ & 44 & 15 & 13 & 12 & 13 & 0 & 2 \\
Saudi Arabia $^{13}$ & 33 & 23 & 21 & 14 & 5 & 0 & 4 \\
Guatemala $^{8}$ & 28 & 10 & 13 & 29 & 0 & 0 & 22 \\
Italy $^{15}$ & 25 & 55 & 5 & 5 & 6 & 0 & 4 \\
USA $^{16}$ & 35 & 45 & 8 & 7 & 4 & 1 & 1 \\
Sudan $^{10}$ & 23 & 48 & 5 & 7 & 6 & 0 & 11 \\
Mexico $^{3}$ & 30 & 9 & 38 & 21 & 1 & 0 & 1 \\
Libya $^{7}$ & 12 & 19 & 24 & 4 & 2 & 1 & 39 \\
\hline
\end{tabular}

Of all patients with CHD, 21 (52.5\%) found to have PAH. PAH in heart disease mainly develops due to high pulmonary blood flow as in left to right shunt lesions.
However, in addition to heart disease, DS itself is a risk factor for PAH. They are at higher risk due to a diminished number of alveoli, a thinner media of pulmonary arterioles and an impaired endothelial function. ${ }^{17}$ King and Tulloh ${ }^{18}$ illustrated in their study that nearly half of children with DS have upper airway obstruction and the same proportion have CHD. Additional problems include pulmonary hypoplasia, structural lung disease and gastro-oesophageal reflux.

The present study showed both single and multiple lesions were associated with PAH. VSD and AVSD both were the most common isolated lesions associated with PAH (12.5\% each) whereas PDA associated lesions were the most common multiple lesions associated with PAH (12.5\%) (Table 2). 5 out of 9 VSD cases $(55.55 \%)$ had PAH while 5 out of 6 AVSD cases (83.33\%) had PAH (Table 3), i.e., though both VSD and AVSD were equally associated with PAH, the proportion of AVSD outnumbered VSD for developing PAH. Literatures ${ }^{19-21}$ are found on DS children with AVSD developing PAH very prematurely. Clapp et al ${ }^{19}$ compared the DS Children with AVSD with normal chromosome children with the same defect regarding development of pulmonary vascular obstructive disease (PVOD). They reviewed their 10 year experiences. Comparisons were made regarding (1) pulmonary blood flow (PBF) and pulmonary vascular resistance (PVR) at initial catheterization, (2) operability as related to elevation in PVR, and (3) age at diagnosis of fixed PVOD. It was found that DS children had significantly lower mean PBF ( $p=0.0001)$ and higher mean PVR $(p=0.0003)$ than normal chromosome counterparts before the age of 1 year. $47 \%$ of the DS children (all ages included) and $80 \%$ of the normal children were considered operable; among inoperable cases due to PVOD, 91.17\% had DS. Fixed PVOD was diagnosed in $12.34 \%$ of DS children before the age of 1 year, while this was found in none of normal children. Hence the author concluded that DS patients with AVSD have a greater degree of elevation of PVR in the first year of life and more rapid progression to fixed PVOD than children with normal chromosomes. In the similar study by Hals et al, ${ }^{20}$ Patients with DS had a higher ratio of pulmonary to systemic vascular resistance than those without DS in the basal situation (in children below1 year of age). It was found that the difference almost disappeared after $100 \%$ oxygen when given to patients with elevated PVR and $11 \%$ of DS patients had fixed elevated PVR. The study concluded that all children with AVSD should be evaluated and operated on at a very young age. Similarly Lange et $\mathrm{al}^{21}$ found that patients with DS exhibited higher preoperative pulmonary artery pressure and resistance compared with patients without DS, however it was concluded that the 
presence of DS per se is not a risk factor for surgical repair of AVSD rather primary repair should be performed within the first 6 months of life because of the early development of pulmonary vascular disease.

In our study, $66.67 \%$ of CHD children already had developed PAH before the age of 1 year (Table 4) which justified that DS children are prone to develop PAH early. Hence it became necessity that the DS children should be evaluated for CHD as soon as possible so that early detection is made and surgical repair can be carried out before the irreversible hemodynamic consequences of pulmonary vascular obstructive disease.

The present study has certain limitations: the study is carried out in the hospital. Though large volume of cardiac patients visited or are being referred here, we cannot extrapolate the result as nation-wise scenario as there are still many cases who are under the tip of ice-berg. They are under diagnosed because either they have not been visited to primary care physician or the socioeconomic status of parents does not allow them to visit Kathmandu from their remote hometown or they died prior to diagnosis. The sample size is small as compared to other similar studies. Cytogenetic studies were not routinely performed on all patients because diagnosis was mainly based on clinical grounds. Diagnosis of PAH is based on echocardiography; had there been data of cardiac catheterization, the proportion of fixed PVOD could have been calculated. Further studies are recommended with larger sample size and cardiac catheterization report. To the best of our knowledge, this is the first study of its kind to be carried out in our centre and in Nepal as well.

\section{Conclusion:}

Congenital heart disease is very common in children with Down syndrome. VSD and AVSD are the two most common cardiac lesions. Children with Down syndrome are likely to develop pulmonary hypertension earlier especially in high left to right shunt cardiac lesions like atrioventricular septal defect. No doubt CHD is the prognostic indicator regarding morbidity and mortality of children with DS. Hence, cardiac screening via echocardiography in all DS children is recommended as soon as possible. Early diagnosis is the key to have proper intervention and to avoid irreversible hemodynamic consequences of pulmonary vascular obstructive disease.

Acknowledgements: Abstract presented at the annual International conference of Cardiac Society of Nepal, Kathmandu, Nepal, November 2-3, 2012.

\section{Conflict of interests: none}

Financial disclosure: none

\section{References:}

1. Stirn Kranjc B. Ocular abnormalities and systemic disease in Down syndrome. Strabismus 2012;20:74-7.

2. Karen S, Brendan L. Down Syndrome and Other Abnormalities of Chromosome Number. In: Kliegman, Behrman, Jenson, Stanton, editors. Nelson textbook of Pediatrics. $19^{\text {th }}$ edition. Philadelphia: Saunders Elsevier; 2007.

3. De Rubens Figueroa J, del Pozzo Magaña B, Pablos Hach JL, et al. Heart malformations in children with Down syndrome. Rev Esp Cardiol 2003;56:894-9.

4. Mohammad A, Khan I, Khan MQ. Hypothyroidism in children with Down's syndrome: A hospital based study. Gomal Journal of Medical Sciences 2012;10:96-9.

5. Chandra N, Cyril C, Lakshminarayana $\mathrm{P}$, et al. Cytogenetic Evaluation of Down Syndrome: A Review of 1020 Referral Cases. Int J Hum Genet 2010;10:8793.

6. Devlin L, Morrison PJ. Accuracy of the clinical diagnosis of Down syndrome. Ulster Med J 2004;73:412.

7. Elmagrpy Z, Rayani A, Shah A, et al. Down syndrome and congenital heart disease: why the regional difference as observed in the Libyan experience? Cardiovasc J Afr 2011;22:306-9.

8. Vida VL, Barnoya J, Larrazabal LA, et al. Congenital cardiac disease in children with Down's syndrome in Guatemala. Cardiol Young 2005;5:286-90.

9. Extracted from Committee on Genetics: Health supervision for children with Down syndrome, Pediatrics 2001;107:442-49 and Baum RA, Spader M, Nash PL, et al: Primary care of children and adolescents with Down syndrome: an update, Curr Prob Pediatr Adolesc Health Care 2008;38:235-68.

10. Ali SK. Cardiac abnormalities of Sudanese patients with Down's syndrome and their short-term outcome. Cardiovasc J Afr 2009;20:112-5.

11. Nisli K, Oner N, Candan S, et al. Congenital heart disease in children with Down's syndrome: Turkish experience of 13 years. Acta Cardiol 2008;63:585-9. 
12. Lo NS, Leung PM, Lau KC, et al. Congenital cardiovascular malformations in Chinese children with Down's syndrome. Chin Med J (Engl). 1989;102:3826.

13. Abbag FI. Congenital heart diseases and other major anomalies in patients with Down syndrome. Saudi Med J 2006;27:219-22.

14. Kava MP, Tullu MS, Muranjan MN, et al. Down syndrome: clinical profile from India. Arch Med Res 2004;35:31-5.

15. Placidi S, Digilio MC, Marino B. Types of cardiac defects in children with Down's syndrome. Cardiol Young 2006;6:198-9.

16. Freeman SB, Taft LF, Dooley KJ, et al. Populationbased study of congenital heart defects in Down syndrome. Am J Med Genet 1998;80:213-7.

17. Vis JC, Duffels MG, Winter MM, et al. Down syndrome: a cardiovascular perspective. J Intellect Disabil Res 2009;53:419-25.

18. King P, Tulloh R. Management of pulmonary hypertension and Down syndrome. Int J Clin Pract Suppl 2011;174:8-13

19. Clapp S, Perry BL, Farooki ZQ, et al. Down's syndrome, complete atrioventricular canal, and pulmonary vascular obstructive disease. J Thorac Cardiovasc Surg 1990;100:115-21.

20. Hals J, Hagemo PS, Thaulow E, et al. Pulmonary vascular resistance in complete atrioventricular septal defect. A comparison between children with and without Down's syndrome. Acta Paediatr 1993;82:595-8.

21. Lange $\mathrm{R}$, Guenther $\mathrm{T}$, Busch $\mathrm{R}$, et al. The presence of Down syndrome is not a risk factor in complete atrioventricular septal defect repair. J Thorac Cardiovasc Surg 2007;134:304-10. 